\title{
Numerical Study of Start Strategy for Ram Accelerator at the Time of Low Injection Pressure
}

\author{
By Ciheng ZHANG and Shiro TAKI \\ Faculty of Engineering, Hiroshima University, Higashi Hiroshima, Japan
}

(Received June 3rd, 2002)

\begin{abstract}
A numerical tool is used to study the role of an ignition tube in the Hiroshima University ram accelerator (named HURAMAC), a unique rectangular shape ram acceleration tube utilized the optical visualization of flow fields. The finite difference method is used to obtain the solution for the reactive gas flow field. An adaptive multi-level grid system is employed for the purpose of effectively using fine mesh points. A detailed methane and oxygen reaction model is considered for the calculation of combustible mixtures. As shown in the simulation results, the process of shock induced ignition and flame propagation is achieved in the ignition tube. After the projectile is moved into the ram acceleration tube where the gas is diluted with a large amount of carbon dioxide, the combustion becomes weak. According to this simulation, it was found that the flame on the projectile surface becomes thinner and the front shock isn't strong enough to pass over the shoulder which is the throat of the supersonic diffuser. The results also show that the flame is held at a stable state in the boundary layer of the projectile surface when burning in a low energetic mixture injected into the ram acceleration tube, successfully starting the ram accelerator at an early stage. The result is a sub-detonative operation mode.
\end{abstract}

Key Words: Ram Accelerator, Shock Wave, Combustion, Flame Propagation

\section{Introduction}

The research of starting processes ${ }^{1,2}$ including shock wave, ignition, combustion and maintaining flame, is fundamental to understanding how a ram accelerator works. When a ram accelerator is to be operated in a sub-detonative mode, heat release behind a projectile is required, and then a thermal choke must be formed to produce strong shock waves around the after-body of the projectile. Here, the key point is to maintain a stable flame on the projectile. To realize a stable thermal choking state behind the projectile in a subdetonative mode, several crucial factors must be adjusted precisely for good performance; for example, the mixture composition, injection pressure, and shape of the projectile and ignition sabot. The former is related to ignition delay time and combustion heat release, and the latter has an influence on the appearance of the flow field and shock waves. According to results using the Hiroshima University ram accelerator, ${ }^{3,4)}$ where low injection pressure is allowed due to the rectangular shape structure, the following shot outcomes were obtained: 1) When an energetic gas mixture is injected, the wave unstart (normal shock moves forward of the projectile) happens in the ram acceleration tube after ignition by sabot impact. 2) When a low energetic gas is employed, the induction time becomes too long to ignite the combustible gas before discarding the ignition sabot. 3) As we know, a large size ignition sabot is very useful for increasing shockwave intensity, thus shortening the ignition time. But the height of the ignition sabot is limited so as to prevent the

(C) 2003 The Japan Society for Aeronautical and Space Sciences production of high-intensity waves, which leads to a reduction in supersonic diffuser performance. In view of the above outcomes, we propose injecting an energetic gas mixture into the ignition tube to facilitate. But a low energetic gas mixture is injected into the next ram acceleration tube to stabilize the flame propagation. This paper discusses a simulation carried out to study how the sub-detonative mode is formed at the early stage of the hot shot in a ram accelerator. Simulation results of ignition and flame propagation in the ignition tube were obtained, ${ }^{5)}$ showing the ignition process in an energetic gas mixture $\left(\mathrm{CH}_{4}+2 \mathrm{O}_{2}+2.5 \mathrm{CO}_{2}\right)$ due to sabot impact within the ignition tube (200 mm long). Shock waves formed depending on heat release at the time of combustion. In the present study, flame stabilization and of thermal choke behavior as the projectile moves from the ignition tube to the ram acceleration tube are investigated.

\section{Calculation Model}

The initial condition is shown in Fig. 1. The tube is divided into three rooms by the diaphragm: a dump tank, an ignition tube, and a ram acceleration tube. The inner height of the tube is $20 \mathrm{~mm}$. The combustible gas mixture $\left(\mathrm{CH}_{4}+2 \mathrm{O}_{2}+2.5 \mathrm{CO}_{2}\right)$ is injected into the ignition tube $(200 \mathrm{~mm}$ long) at $0.30 \mathrm{MPa}$, and the gas mixture $\left(\mathrm{CH}_{4}+2 \mathrm{O}_{2}+5.5 \mathrm{CO}_{2}\right)$ is injected into the ram acceleration tube at $0.45 \mathrm{MPa}$. The temperature of both gas mixtures is $293.15 \mathrm{~K}$. It is assumed that the diaphragm is completely broken down when the sharp wedge of the projectile contacts it. Initially, a projectile and an ignition sabot move from the 


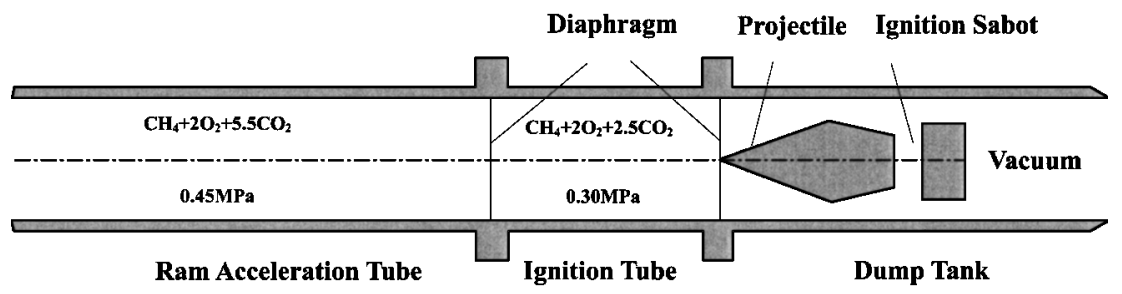

Fig. 1. The initial condition of the present simulation.

dump tank (vacuum) at a velocity of $1,100 \mathrm{~m} / \mathrm{s}$. Based on the gas mixture in the ignition tube, the Mach number of the projectile is 3.6. The projectile has a leading wedge angle of $22^{\circ}$, a shoulder height of $13 \mathrm{~mm}$, a length of $53 \mathrm{~mm}$, and weight of $6 \mathrm{~g}$. The simulation begins when the projectile nose penetrates the diaphragm between the dump tank and ignition tube.

The boundary layer on the projectile surface is considered as well, so a non-slip condition is employed at the interface between the fluid and the projectile. Although the flow speed varies along the tube wall, a slip condition is assumed for the wall surface of the tube. The velocity difference between the wall and fluid is not that large when compared to that of the projectile surface because the flow is expected to speed up at the after-body of projectile, and even expand in the combustion region. An adiabatic condition is used for all object surfaces, neglecting the heat exchange from the gas mixture to the object.

Corresponding to the typical conditions during experiments utilizing the Hiroshima University ram accelerator, a methane and oxygen chemical reaction model is considered. Here the chain reaction model proposed by Seshadri and Peters ${ }^{6)}$ is employed for calculating the chemical reaction term. This model is composed of 35 reaction steps, and 15 chemical species are included.

\section{Governing Equations}

The basic equations used in the present computation contain the compressible Navier-Stokes equations. The conservation equations are written in vector form as:

1) The conservation of mass

$$
\frac{\partial \rho}{\partial t}+\nabla \cdot(\rho \boldsymbol{v})=0 .
$$

2) The conservation of momentum

$$
\frac{\partial \boldsymbol{v}}{\partial t}+\boldsymbol{v} \cdot \nabla \boldsymbol{v}=-\frac{1}{\rho}(\nabla \cdot \boldsymbol{P}) .
$$

3) The conservation of energy

$$
\rho \frac{\partial e}{\partial t}+\rho \boldsymbol{v} \cdot \nabla e=-\nabla \cdot \boldsymbol{q}-\boldsymbol{P}:(\nabla \boldsymbol{v}) .
$$

4) The conservation of chemical species

$$
\frac{\partial Y_{i}}{\partial t}+\boldsymbol{v} \cdot \nabla Y_{i}=w_{i}-\frac{1}{\rho}\left[\nabla \cdot\left(\rho Y_{i} \boldsymbol{V}_{i}\right)\right]
$$

Where $e, \boldsymbol{P}, \rho$, and $\boldsymbol{v}$ denote the specific total energy, pres- sure tensor, density and velocity vector, respectively. $Y_{i}, \boldsymbol{V}_{i}$, and $w_{i}$ are the mass fraction, diffusion velocity vector and production rate of species $i$, respectively.

Neglecting thermal diffusion and pressure diffusion, the binary diffusion of species is considered:

$$
\sum_{j=1}^{N} \frac{X_{j}}{D_{i j}}\left(\boldsymbol{V}_{i}-\boldsymbol{V}_{j}\right)=-\frac{\nabla X_{i}}{X_{i}}, \quad(i=1, \cdots, N),
$$

where $X_{i}$ is the molar fraction of species $i$ and $D_{i j}$ is the diffusion coefficient between species $i$ and $j$.

Taking into account only shear stress, the pressure tensor is expressed as:

$$
\boldsymbol{P}=\left[p+\frac{2}{3} \mu \nabla \cdot \boldsymbol{v}\right] \boldsymbol{U}-\mu\left[(\nabla \boldsymbol{v})+(\nabla \boldsymbol{v})^{\mathrm{T}}\right],
$$

where $\mu$ is the viscosity coefficient, $p$ is the pressure, and $\boldsymbol{U}$ is the unit tensor.

Neglecting the radiation heat transfer, the heat flux equation becomes:

$$
\boldsymbol{q}=-\lambda \nabla T+\rho \sum_{i=1}^{N} h_{i} Y_{i} \boldsymbol{V}_{i}
$$

where $\lambda$ is the heat conduction coefficient of the gas mixture.

The molecular transport coefficients for each species $i$ are approximated as functions of the temperature, which are basically estimated by the Chapman-Enskog formula ${ }^{7)}$ :

$\rho D_{i j} \approx D_{i j}^{o} \cdot T^{0.668}, \mu_{i} \approx \mu_{i}^{o} \cdot T^{0.653}, \lambda_{i} \approx \mu_{i}\left(c_{p i}+\frac{5}{4} R_{i}\right)$.

In the present calculation, a thermally perfect gas is assumed:

$$
p=\rho R^{o} T / \bar{M}=\rho R^{o} T \sum_{i=1}^{N} Y_{i} / M_{i}
$$

But the enthalpies are approximated by polynomials of the temperature:

$$
\frac{h_{i}}{R T}=\left[a_{i 1}+\frac{a_{i 2}}{2} T+\frac{a_{i 3}}{3} T^{2}+\frac{a_{i 4}}{4} T^{3}+\frac{a_{i 5}}{5} T^{4}+\frac{a_{i 6}}{T}\right] .
$$

\section{Numerical Methods}

The finite difference method is used for resolving the Navier-Stokes equations, including chemical reaction term. The explicit McCormack two-step method is used for calculation of the hyperbolic equation. The alternating direction 
implicit technique is used for the heat conduction term, and the diffusion and viscosity terms are integrated using an explicit two-step method. The chemical reaction term is solved at each grid point using the implicit Lomax-Baily method.

The square grids are arranged for discrete points throughout the computational domain. The adaptive multilevel grid refinement method is applied for grid generation to improve numerical accuracy. The basic mesh size is $1 \mathrm{~mm}$ on the lowest grids, and the size is decreased to $1 / 3$ of the coarse mesh after a single refinement upgrade. In the present simulation, fine meshes $(37 \mu \mathrm{m})$ are generated in the area of the flame and boundary layer, according to the concentration of chemical species and velocity gradient.

\section{Results and Analysis}

When performing the present simulation, the injection pressure in the tubes, premixed gas mixture and initial condition of the flow field are set corresponding to a previous HURAMAC experiment. The injection pressures of the gases in the evacuation tube, ignition tube and ram acceleration tube are $120 \mathrm{~Pa}, 0.3 \mathrm{MPa}$, and $0.45 \mathrm{MPa}$, respectively. The simulation begins when the projectile moves into the ignition tube, where a $\mathrm{CH}_{4}+2 \mathrm{O}_{2}+2.5 \mathrm{CO}_{2}$ mixture is injected. The simulation results are shown in the time sequence of a combustion flow field with the origin of coordinate axes set on the wedge head of the projectile.

\subsection{The first stage: Sabot impact ignition in the igni- tion tube}

The simulation results are presented as the distribution of $\mathrm{CH}_{4}$ mass fraction in Fig. 2. It was found that at $60 \mu \mathrm{s}$ after the projectile moves into the ignition tube, an ignition source is formed before the ignition sabot. Due to the impact of the sabot, a detached shock is produced to speed up the induction chemical reaction. Then the flame propagates forward in the vortex stream that exists in the recirculation area between the projectile and the sabot. At $120 \mu \mathrm{s}$, the flame contacts the base surface of the projectile, and is then maintained on the projectile surface. It can be said that the $200 \mathrm{~mm}$ long ignition tube in the Hiroshima University ram accelerator is proper for the purpose of ignition when a $\mathrm{CH}_{4}+2 \mathrm{O}_{2}+2.5 \mathrm{CO}_{2}$ mixture is used as the combustible gas.

\subsection{The second stage: Moving into the ram accelera-} tion tube

After the diaphragm between the ignition tube and ram acceleration tube is ruptured, the projectile moves into the ram acceleration tube, into which a $\mathrm{CH}_{4}+2 \mathrm{O}_{2}+5.5 \mathrm{CO}_{2}$ mixture is injected. The distributions of the $\mathrm{CH}_{4}$ mass fraction are shown in Fig. 3, which presents the combustion field and interface of the mixtures in the ignition tube and ram acceleration tube. At the time of $180 \mu \mathrm{s}$, the projectile head contacts the low energetic gas mixture in the ram acceleration tube. Because the energetic gas is still burnt behind the shoulder of the projectile, a large amount of heat is released to support wide flame propagation. At the time of $210 \mu \mathrm{s}$, the projectile shoulder moves into the ram acceleration tube, and then the combustion occurs in the low energetic gas. As the burning rate decreases, the flame near the shoulder becomes thinner (comparison of the results from $210 \mu$ s to $240 \mu \mathrm{s}$ ), showing weakening of the combustion state. At the time of $240 \mu \mathrm{s}$, the whole body of the projectile has entered the ram acceleration tube. It is seen that a stable flame is maintained on the boundary layer of the projectile.

When a mixture of hydrogen and oxygen diluted by nitrogen was used in the simulation, ${ }^{4)}$ the detonation wave formed quickly after ignition. Therefore, the problem to solve was how to change the detonation to deflagration before it moves forward of the projectile. According to the present simulation, a different result is obtained when a $\mathrm{CH}_{4}-\mathrm{O}_{2}-\mathrm{CO}_{2}$ mixture is used. Only propagation of the deflagration wave is found in the ignition tube. The adjustment of mixture composition has to be performed in the ram acceleration tube to control the burning velocity at a satisfactory level.

The time sequence of pressure is shown in Fig. 4. At the wedge of the projectile, well-known oblique shock and its reflection shock are formed in the supersonic diffuser. From the shoulder of the projectile, an expansion wave is formed.

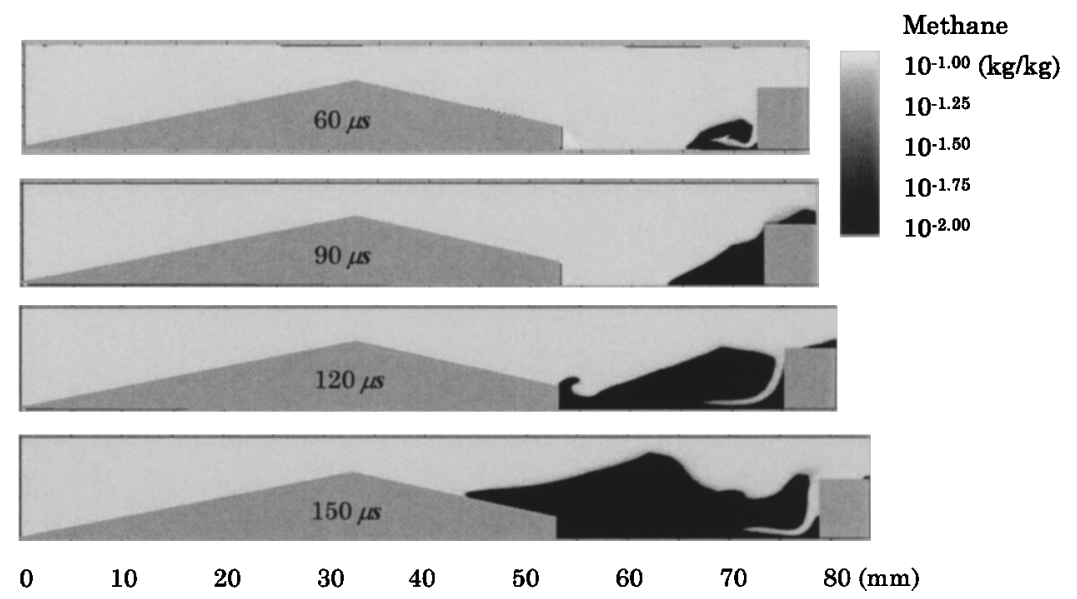

Fig. 2. Time sequence of the distribution of $\mathrm{CH}_{4}$ mass fraction; when the projectile is moving in the ignition tube, showing the ignition and the combustion development. 


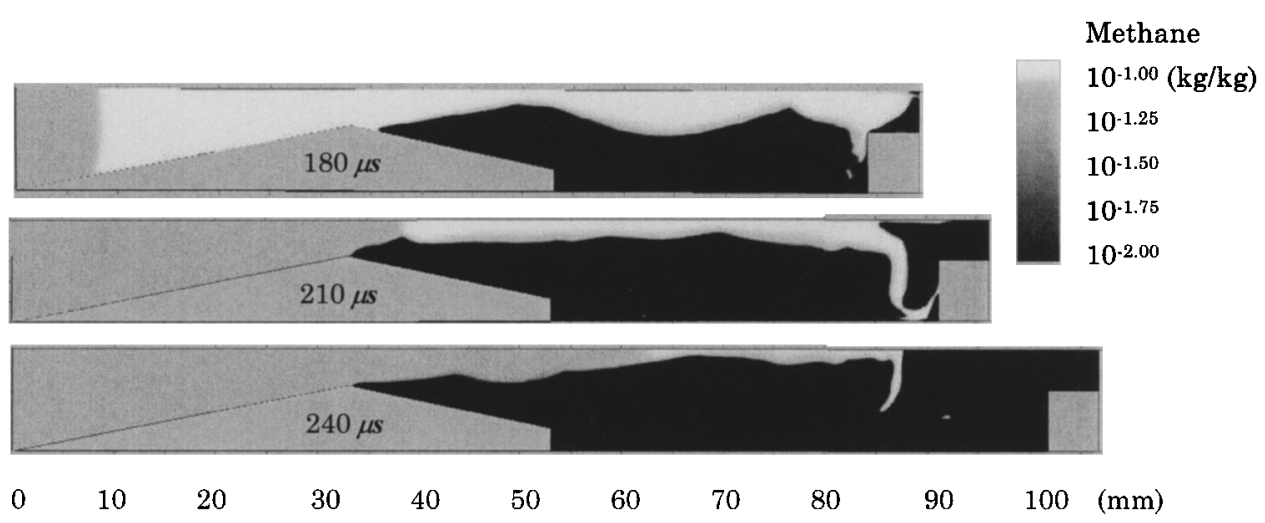

Fig. 3. Time sequence of the distribution of $\mathrm{CH}_{4}$ mas fraction; when the projectile moves in the ram acceleration tube.
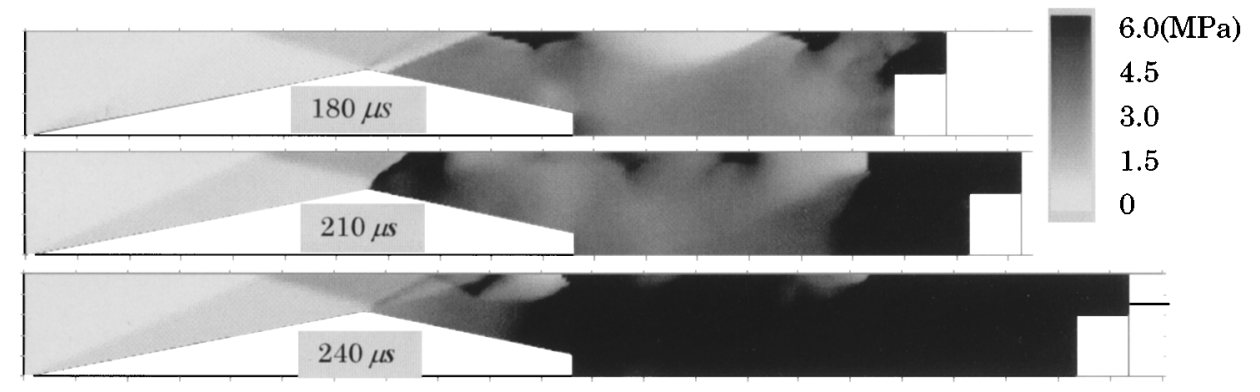

$\begin{array}{llllllllllll}0 & 10 & 20 & 30 & 40 & 50 & 60 & 70 & 80 & 90 & 100 & (\mathrm{~mm})\end{array}$

Fig. 4. Time sequence of the pressure distribution; when the projectile moves in the ram acceleration tube.

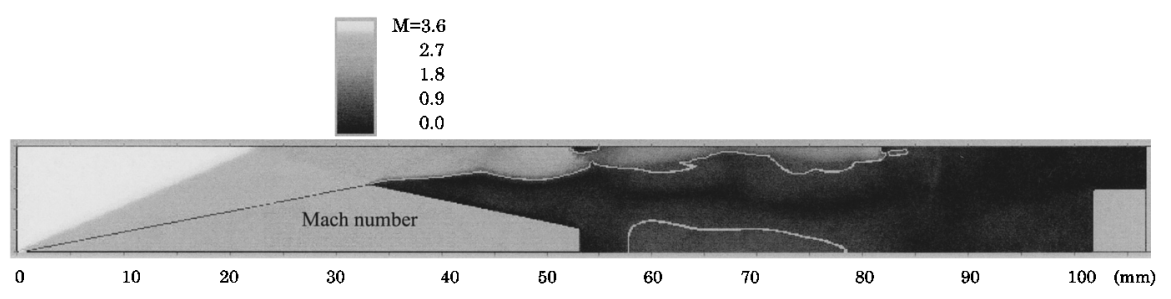

Fig. 5. Distribution of Mach number; where the white lines represent the sonic velocity at the time of $240 \mu \mathrm{s}$.

But it doesn't expand far because oblique shock is produced in front of the flame, which is propagating to a location near the shoulder. After the shock wave hits the wall, high pressure is formed near the tube. It was also found that strong shock waves are produced before the ignition sabot due to a choking effect. Until $210 \mu \mathrm{s}$, the oblique shock is moves forward with the propagation of flame. But it is clearly shown that this shock isn't strong enough to become normal shock. This prevents the choke from being formed at the throat. After the projectile has moved in the ram acceleration tube at $240 \mu \mathrm{s}$, the oblique shock near the shoulder becomes weak and recedes from the throat. At this time, the pressure at the base of the projectile increases rapidly, producing large thrust on the projectile.

As remarked by Hertzberg et al., ${ }^{8)}$ when a ram accelerator is operated in the sub-detonative mode, subsonic flow is formed before the choking point. In the present simulation results of Fig. 5, in which the white line indicates the sonic velocity line, we see that, after the oblique shock waves, there is a remarkable decrease in the Mach number of the fluid to subsonic value when the gas flows into the combustion area. This highlights the choking effect on the gas mixture upstream, although the choke point isn't shown in this result due to computation domain limitations. At the time of $240 \mu \mathrm{s}$, the ignition sabot is being discarded, so we can say that the thermal choke gradually occurs in the process of forming a sub-detonative mode.

Separation of the boundary layer at the after-body of the projectile is shown in Fig. 6, where the flow directions are represented by the arrows. When the boundary layer becomes very thick in the separation area, the flame can be suitably held. In Fig. 7, the flow field downstream of the projectile is shown. It can be seen that a group of vortices is formed in the flame. In the upper area of the vortices, the gas flows downstream, but in the recirculation region, it flows upstream. According to the pressure results shown in Fig. 4, at the time of $240 \mu \mathrm{s}$, it can be explained that the turbulence in the stream enhances the combustion and therefore 


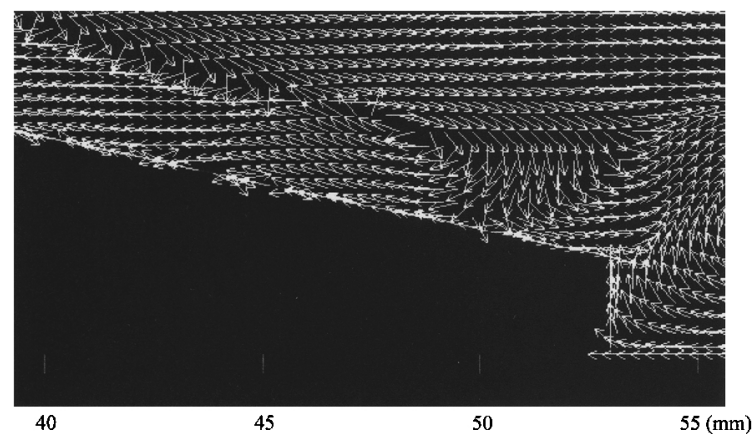

Fig. 6. Flow field around the after-body of projectile at the time of $200 \mu \mathrm{s}$; where the flow direction is represented by the arrows.

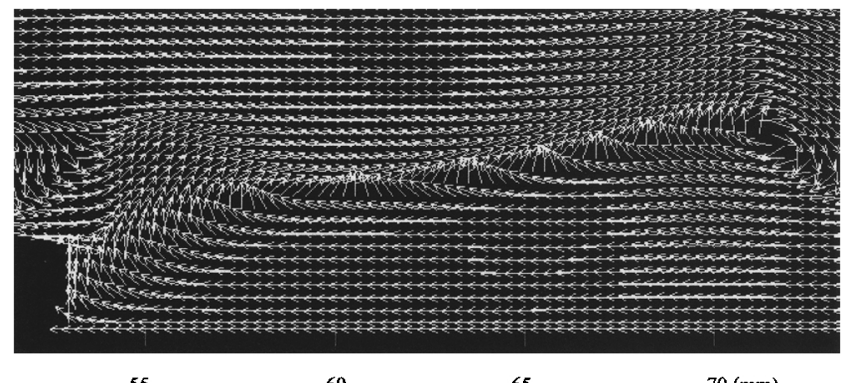

55

65

$70(\mathrm{~mm})$

Fig. 7. Flow field behind the base of projectile at the time of $200 \mu \mathrm{s}$ where the flow direction is represented by the arrows.

increases the pressure. In the present simulation, although the ignition sabot doesn't move far, the increased heat release leads to thermal choking downstream, and then produces thrust on the projectile.

\section{Conclusion}

Using an ignition tube for the purpose of quick ignition is proposed using the Hiroshima University ram accelerator. A simulation was conducted for a projectile moving from the ignition tube to the ram acceleration tube. We found that an ignition source is produced by sabot impact and then the flame propagates to the projectile. In the ignition tube, the flame is maintained on the projectile surface and propagates to the shoulder along the boundary layer. After moving into the ram acceleration tube, the shock isn't strong enough to become normal shock, preventing a choking condition from occurring at the throat. Through this simulation, we have shown that an ignition tube is useful for quick ignition. A sub-detonative mode is gradually established with flame stabilization when a projectile is moved into the ram acceleration tube.

\section{References}

1) Schultz, E., Knowlen, C. and Bruckner, A. P.: Overview of the Sub-detonative Ram Accelerator Starting Process, Ram Accelerators, Springer-Verlag, Berlin Heidelberg, 1998, pp. 189-203.

2) Taki, S., Zhang, C. and Chang, X.: Numerical Simulation of the Unsteady Process in Starting Period of Ram Accelerator, Ram Accelerators, Springer-Verlag, Berlin Heidelberg, 1998, pp. 215-221.

3) Chang, X., Matsuoka, S., Watanabe, T. and Taki, S.: Ignition Study for Low Pressure Combustible Mixture in a Ram Accelerator, Ram Accelerators, Springer-Verlag, Berlin Heidelberg, 1998, pp. 105-109.

4) Zhang, C., Taki, S. and Chang, X.: Numerical Simulation of the Starting Process of the Two-Dimensional Ram Accelerator with an Ignition Tube, J. JSME B, 64 (1998), pp. 2655-2661.

5) Zhang, C. and Taki, S.: Numerical Study of Shock Induced Ignition and Combustion of $\mathrm{CH}_{4}-\mathrm{O}_{2}-\mathrm{CO}_{2}$ Mixtures in a Two Dimensional Ram Accelerator, J. Physique IV France, 10 (2000), pp. 155-164.

6) Seshadri, K. and Peters, N.: The Inner Structure of Methane-Air Flames, Combust. Flame, 81 (1990), pp. 96-118.

7) Hirschfelder, J. O., Curtiss, C. F. and Bird, R. B.: Molecular Theory of Gases and Liquids, John Wiley \& Sons, New York, 1964.

8) Hertzberg, A., Bruckner, A. P. and Bogdanoff, D. W.: Ram Accelerator: A New Chemical Method for Accelerating Projectile to Ultrahigh Velocities, AIAA J., 26 (1988), pp. 195-203. 